\title{
A case of carcinoma in an adenoma of the duodenal minor papilla successfully treated with endoscopic mucosal resection
}

Authors

Institutions
Toru Matsui ${ }^{1}$, Hiroyuki Matsubayashi ${ }^{1}$, Kinichi Hotta ${ }^{1}$, Keiko Sasaki $^{2}$, Hiroaki Ito ${ }^{3}$, Hiroyuki Ono ${ }^{1}$

${ }^{1}$ Division of Endoscopy, Shizuoka Cancer Center, Nagaizumi, Suntogun, Shizuoka, Japan

${ }^{2}$ Division of Pathology, Shizuoka Cancer Center, Nagaizumi, Suntogun, Shizuoka, Japan

${ }^{3}$ Division of Diagnostic Radiology, Shizuoka Cancer Center, Nagaizumi, Suntogun, Shizuoka, Japan submitted 8. August 2015 accepted after revision 14. December 2015

\section{Bibliography}

Dol http://dx.doi.org/

10.1055/s-0041-111500

Published online: 10.2.2016

Endoscopy International Open 2016; 04: E252-E254

(c) Georg Thieme Verlag KG

Stuttgart · New York

E-ISSN 2196-9736

\section{Corresponding author} Hiroyuki Matsubayashi, MD, PhD

Division of Endoscopy Shizuoka Cancer Center 1007 Shimonagakubo Nagaizumi, Suntogun, Shizuoka, 411-8777

Japan

Phone: +81-55-989-5222

Fax: +81-55-989-5222

h.matsubayashi@scchr.jp
Background and study aims: Endoscopic papillectomy is currently used to treat noninvasive tumors of the papilla of Vater, but it is seldom reported for treatment of similar tumors of the minor papilla. This report describes the case of a 69year-old female with a tumor located at the duodenal minor papilla. Findings of duodenoscopy, biopsy, and pancreatography indicated that her noninvasive tumor of the minor papilla was suitable for treatment with endoscopic resection. Glycerol-injected endoscopic mucosal resection (EMR) was performed, and the resected material

\section{Introduction}

\section{$\nabla$}

Tumors of the duodenal minor papilla are very rare. In contrast to tumors of the major papilla, those of the minor papilla are less symptomatic unless they grow large or are accompanied by pancreatic duct anomalies [1]. Consequently, such tumors are incidental findings made during health screenings. As with other duodenal lesions, most tumors of the minor papilla are treated by laparotomy, including pancreatoduodenectomy, papillectomy, and duodenectomy [13]. In cases of adenoma or dysplasia located at the major papilla, endoscopic papillectomy (EP) is the least invasive and curative procedure when the neoplasm is limited to the duodenum and common channel $[4,5]$. However, to date, only a few cases of endoscopic resection have been reported for noninvasive tumors of the minor papilla, which differ in anatomic characteristics from those of the major papilla.

\section{Case report}

$\nabla$

A 69-year-old woman was referred to our hospital for investigation and treatment of a duodenal tumor. Laboratory data, including tumor markers and pancreatic enzymes, were all within normal histologically showed carcinoma in the adenoma, negative for neoplastic extension at the cut margin. No complications occurred during the treatment course, and no recurrence has been recognized for 80 months. Unlike the major papilla of Vater, the minor papilla can be lifted up by submucosal injection. Noninvasive epithelial tumors of the duodenal minor papilla without extension to the pancreatic duct can be successfully treated with EMR, as the technique is easy, it is minimally invasive, and it is curative.

ranges. On referral, endoscopic examination demonstrated a slightly elevated, multilobular tumor at the minor papilla of the duodenum, approximately $12 \mathrm{~mm}$ in size. Under sprinkling indigo carmine pigment, no ulceration, depressed areas, or large nodules suggestive of an invasive component were evident macroscopically ( $\bullet$ Fig. 1 a,b). The major ampulla of Vater was normal ( $\bullet$ Fig.1c). Endoscopic retrograde cholangiopancreatography (ERCP) revealed no obvious tumor extension to the Santorini duct ( Fig. 2). No anomaly of the pancreatobiliary ductal system was recognized and the contrast medium was fully excreted from the major papilla after withdrawal of the catheter. A biopsy from the minor papilla demonstrated histology of adenoma with severe atypia (or high-grade dysplasia). Based on these findings, we diagnosed a tumor limited to the mucosal layer of the minor papilla with a good indication for endoscopic mucosal resection (EMR).

EMR was performed the day after admission. Glycerol was first injected into the submucosa to separate the muscularis propria, facilitate easier resection, and prevent perforation ( $\bullet$ Fig. 1 d). A well-elevated lesion was ligated and cut using an electric snare (Captivator, Boston Scientific, Alajuela, Costa Rica) with an endocut mode of electrification (cutting at maximum: 120 watt, ERBE ICC 200, Marietta, U.S.A) ( $\bullet$ Fig. 1 e). A resected 

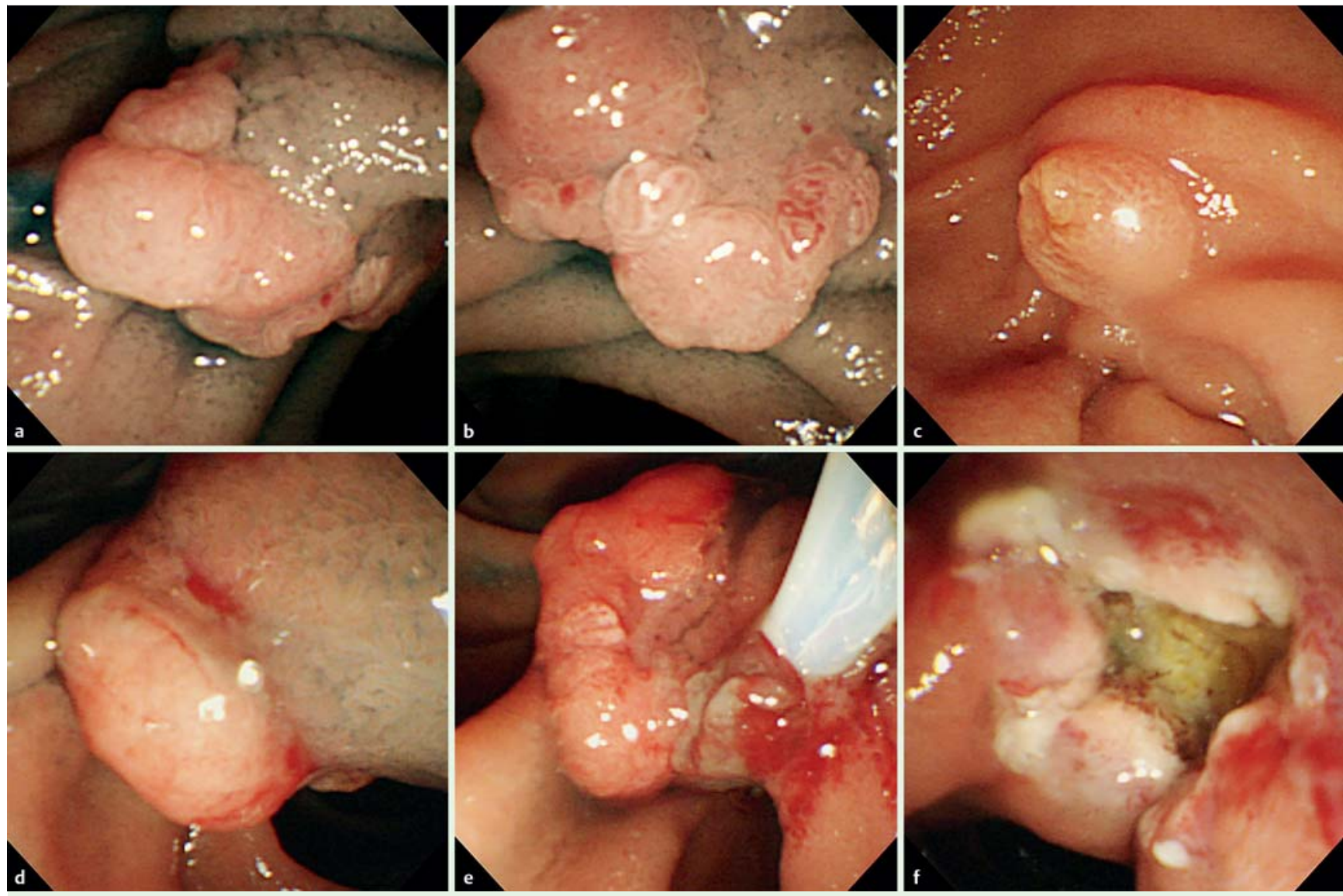

Fig. 1 Side-viewing endoscope view of a tumor of the minor duodenal papilla. a Oral-side view. $\mathbf{b}$ Anal-side view. $\mathbf{c}$ Intact orifice of the major papilla. d A glycerol-injected and well-lifted tumor. e Electric snare ligation. $\mathbf{f}$ An ulceration after endoscopic mucosal resection (EMR) without remnant tumor.

sample was collected using a net (Roth Net ${ }^{\circledR}$, US endoscopy, Ohio, U.S.A). Finally, the ulceration after the EMR was confirmed to be negative for any remnant lesion, bleeding, or perforation ( $\bullet$ Fig. 1f). The endoscopic procedure was completed without stent placement to the minor papilla. No pancreatitis or other complications occurred related to the endoscopic procedure. The patient began eating on the second day after EMR and she was discharged as scheduled.

Histology of the EMR material showed an adenocarcinoma (carcinoma in situ) component within the adenoma $(11 \times 8 \mathrm{~mm})$ and all cut ends were negative for any neoplastic extension ( $\bullet$ Fig. 3). For post-EMR surveillance, upper gastrointestinal endoscopy was continued, first at 6-months intervals and then annually. Eighty months have passed since the EMR and the patient remains healthy with no evidence of recurrence, increasing level of serum amylase, or dilation of the pancreatic duct.

\section{Discussion}

A variety of tumors have been reported at the minor papilla of the duodenum, including endocrine tumors, adenomyomas, and adenocarcinomas. Most of these cases have been treated by laparotomy [ $1-3$ ]. Early-stage tumors of the major papilla (although the indication is still arguable $[4,5]$ ) are increasingly treated by endoscopic resection with relative ease and low invasiveness. However, to date, only a few cases of endoscopic resection have been reported for the tumors of the minor papilla $[6,7]$. There-

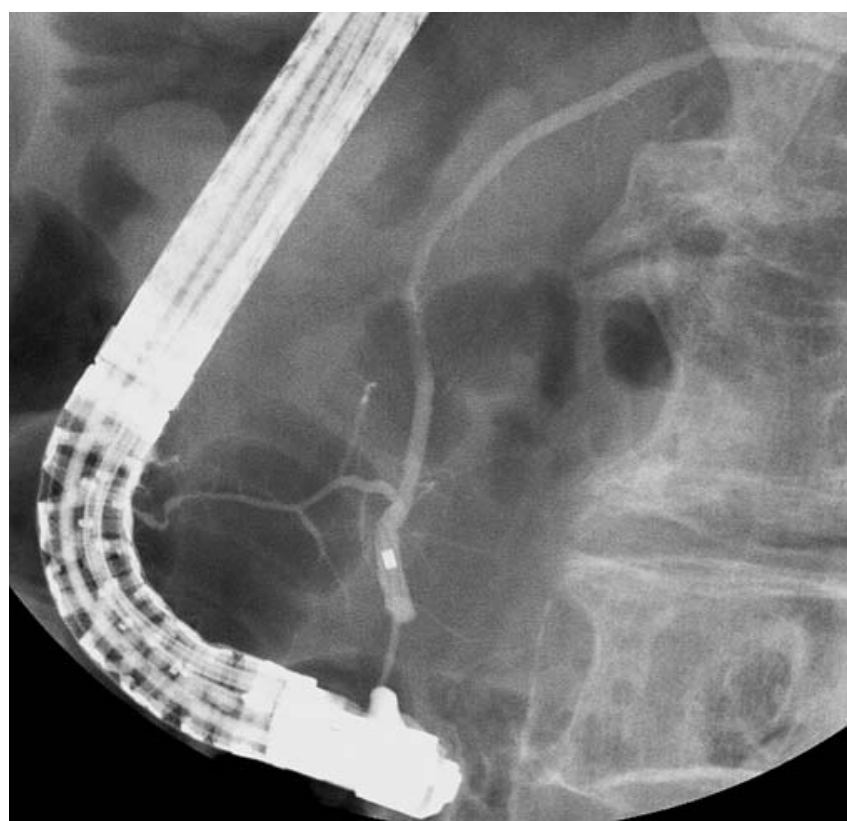

Fig. 2 Endoscopic retrograde pancreatography by cannulation from the major papilla demonstrating intact Wirsung and Santorini ducts without pancreatobiliary duct malformations. 

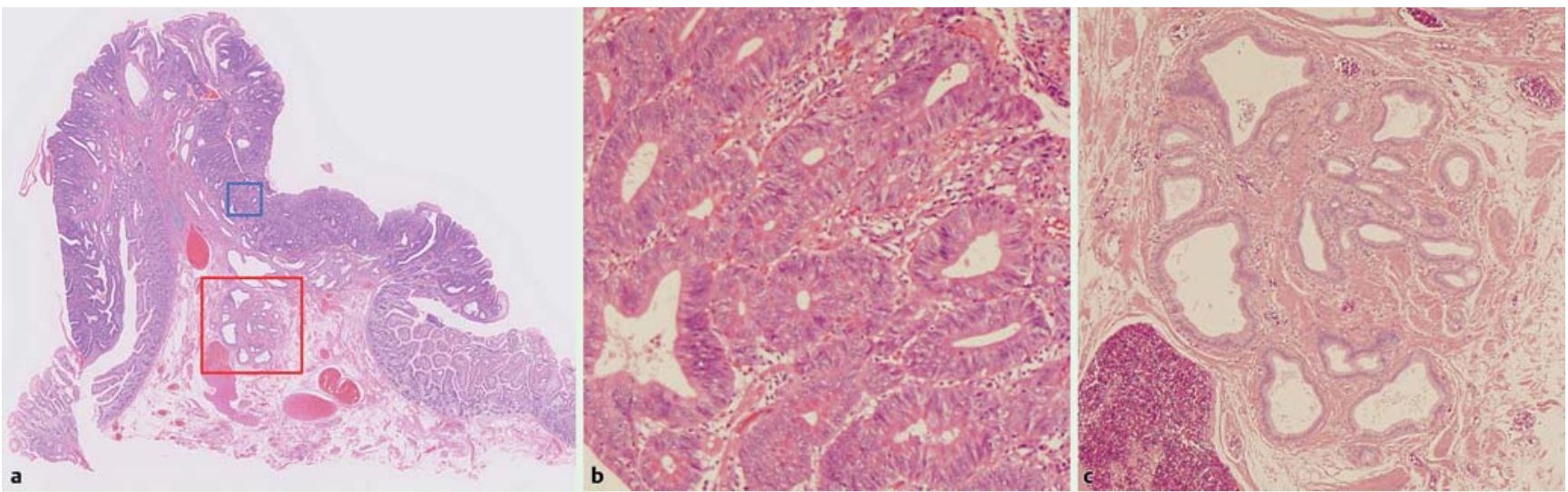

Fig. 3 Histology of the EMR sample of the minor papilla. a A low-power view of the EMR specimen showing a flat-elevated epithelial lesion of the minor papilla. b A magnified view of the orifice epithelia histologically showing adenocarcinoma in situ accompanied by a high-grade adenoma component. $\mathbf{~ A ~ h i g h ~}$ power view of the Santorini duct without extension of the neoplastic cells.

fore, no consensus exists regarding the indication and method of endoscopic treatment.

Ampullary adenoma without ductal extension into the bile/pancreatic duct is currently considered an indication for EP for tumors of the major papilla $[4,5,8]$. Containment of the adenoma and limitation within the ampullary duodenum and common duct suggest the so-called intestinal-type, a noninvasive tumor of the papilla of Vater [9]. However, application of this indication to the minor papilla requires that we bear in mind the differences in anatomic characteristics of the two duodenal papillae. First, the minor papilla is usually not a main exit for pancreatic juice secretion, suggesting a lower risk of post-resection pancreatitis if the pancreatic duct's anomaly does not exist and the major papilla is intact. In this sense, Trevino et al. reported an interesting case of minor papilla adenoma accompanied with pancreatic divisum and successfully treated by endoscopic resection with placement of a plastic stent to the dorsal pancreatic duct [7]. In another case in their series, tubulovillous adenoma was confirmed at both major and minor papilla, and they successfully resected both of these lesions at one session with a stent insertion to the Wirsung duct as prophylaxis for post-procedure pancreatitis [7]. Second, the surrounding muscular construction is not as massive for the minor papilla as it is for the major papilla [10]. Hence, unlike the case for the major Vater, a lesion can be lifted significantly by submucosal injection, which probably enabled an easier en-bloc resection in the current case, even of a flat elevated lesion, with less risk of bleeding or perforation. One concern is that the minor papilla often contains pancreatic tissue (80\%) [10] and the area lined by the pancreas may not be as wide as that of the major Vater. Therefore, risks of perforation may increase in cases of laterally spreading tumors resected without submucosal injection.

With epithelial neoplasms of the major and minor papillae, evaluation of the intraductal extension of the neoplastic cells often is problematic and it is sometimes difficult even when using endoscopic ultrasonography (EUS) or intraductal ultrasonography (IDUS) $[4,5]$. The operator can judge ductal extension as positive if it is recognized as a millimeter level of neoplastic component in the bile/pancreatic duct by either EUS or IDUS. However, a single layer of neoplastic cell extension is $<20 \mu \mathrm{m}$ thick and cannot be distinguished with clinical imaging. In our case, we denied the macroscopic level of Santorini duct extension by ERP ( $\bullet$ Fig. 2 ) and planned to add laparotomy if the carcinoma was positive, even at the microscopic level, at the cut end of the pancreatic duct $(\bullet$ Fig. 3c).

EMR is considered a good treatment option for noninvasive tumors of the duodenal minor papilla that show no intraductal extension to the dorsal pancreatic duct. Experience with more of these types of cases is needed to reach consensus regarding the optimal method for diagnosing and treating tumors of the duodenal minor papilla.

\section{Competing interests: None}

\section{References}

1 Yamao K, Ohhashi K, Furukawa T et al. Primary carcinoma of the duodenal minor papilla. Gastrointest Endosc 1998; 48: 634-636

2 Fukuda A, Yazumi S, Sawada M et al. Adenomyoma of the minor duodenal papilla. Gastrointest Endosc 2005; 61: 475-479

3 Wang HY, Chen MJ, Yang TL et al. Carcinoid tumor of the duodenum and accessory papilla associated with polycythemia vera. World J Gastroenterol 2005; 11: 3794-3796

4 Chini P, Draganov PV. Diagnosis and management of ampullary adenoma: The expanding role of endoscopy. World J Gastrointest Endosc 2011; 3: $241-247$

5 Ito K, Fujita N, Noda Y. Endoscopic diagnosis and treatment of ampullary neoplasm (with video). Dig Endosc 2011; 23: 113-117

6 Sugiyama M, Kimura W, Muto T et al. Endoscopic resection of adenoma of the minor papilla. Hepatogastroenterology 1999; 46: 189-192

7 Trevino JM, Wilcox CM, Varadarajulu S. Endoscopic resection of minor papilla adenomas (with video). Gastrointest Endosc 2008; 68: $383-$ 386

8 Binmoeller KF, Boaventura S, Ramsperger K et al. Endoscopic snare excision of benign adenomas of the papilla of Vater. Gastrointest Endosc 1993; 39: $127-131$

9 Matsubayashi H, Watanabe H, Yamaguchi T et al. Differences in mucus and K-ras mutation in relation to phenotypes of tumors of the papilla of vater. Cancer 1999; 86: 596-607

10 Suda K. Histopathology of the minor duodenal papilla. Dig Surg 2010; 27: $137-139$ 